\title{
Effectiveness of eHealth Interventions for HIV Prevention and Management in Sub-Saharan Africa: Systematic Review and Meta-analyses
}

\author{
Louisa Manby ${ }^{1} \cdot$ Catherine Aicken $^{2,3} \cdot$ Marine Delgrange $^{1} \cdot$ Julia V. Bailey $^{4}$ (I)
}

Accepted: 23 July 2021 / Published online: 24 August 2021

(c) The Author(s) 2021

\begin{abstract}
HIV is still the leading cause of death in Sub-Saharan Africa (SSA), despite medical advances. eHealth interventions are effective for HIV prevention and management, but it is unclear whether this can be generalised to resource-poor settings. This systematic review aimed to establish the effectiveness of eHealth interventions in SSA. Six electronic databases were screened to identify randomised controlled trials (RCTs) published between 2000 and 2020. Meta-analyses were performed, following Cochrane methodology, to assess the impact of eHealth interventions on HIV-related behaviours and biological outcomes. 25 RCTs were included in the review. Meta-analyses show that eHealth interventions significantly improved HIV management behaviours (OR 1.21; 95\% CI 1.05-1.40; $\mathrm{Z}=2.67 ; \mathrm{p}=0.008$ ), but not HIV prevention behaviours (OR 1.02; $95 \%$ CI $0.78-1.34 ; Z=0.17 ; p=0.86$ ) or biological outcomes (OR 1.17; 95\% CI 0.89-1.54; $Z=1.10 ; p=0.27$ ) compared with minimal intervention control groups. It is a hugely important finding that eHealth interventions can improve HIV management behaviours as this is a low-cost way of improving HIV outcomes and reducing the spread of HIV in SSA. PROSPERO registration number: CRD42020186025.
\end{abstract}

Keywords Human immunodeficiency virus $\cdot$ eHealth $\cdot$ Sub-Saharan Africa $\cdot$ Systematic review $\cdot$ Meta-analysis

\section{Background}

Around 70\% of global HIV diagnoses are in Sub-Saharan Africa (SSA), where sexual transmission remains the primary mode of HIV transmission [1]. Despite recent medical advances, HIV is still the leading cause of death in the region $[1,2]$. Poor outcomes among those living with HIV in SSA are linked to delayed diagnosis, poor linkage to HIV care and inconsistent adherence to anti-retroviral therapies

Julia V. Bailey

julia.bailey@ucl.ac.uk

1 Institute of Epidemiology and Healthcare, University College London, London, UK

2 School of Health Sciences, University of Brighton, Falmer, Brighton, UK

3 Centre for Population Research in Sexual Health and HIV, Institute for Global Health, University College London, London, UK

4 eHealth Unit, Department of Primary Care and Population Health, University College London (Royal Free Campus), Rowland Hill Street, London NW3 2PF, UK
[3]. More than half of people living with HIV in SSA are unaware of their HIV status and $\sim 60 \%$ are not receiving treatment [4]. Given SSA's high disease burden and extreme shortage of healthcare workers, there remains an unmet need for interventions which deliver tailored support for the prevention and management of sexually transmitted HIV in the region and digital health strategies have great potential to meet this need [5].

Information and communications technology use has been rapidly increasing since the early 2000 s in SSA $[6,7]$. The majority of the SSA population own a mobile phone and ownership is now as high as $80 \%$ in some countries [8]. Basic phones are the most common mobile device owned in SSA and the likelihood of ownership is related to income and level of education [8]. With increasing technology use, eHealth in SSA has expanded over the last $\sim 20$ years and there is great enthusiasm for its potential for improving health outcomes $[9,10]$. Improving HIV/AIDS outcomes has been a major focus of digital health strategies in SSA [11]. These strategies include mobile phone-based reminders and messages aimed at improving ART medication adherence and attendance to medical appointments as well as 
providing health information related to HIV treatment and prevention [11].

Systematic reviews have demonstrated the effectiveness of eHealth interventions for HIV prevention and management [12-14], but it is unclear whether findings can be generalised to resource-poor settings $[9,10]$. As such, this study aimed to systematically evaluate the effectiveness of eHealth interventions for sexually transmitted HIV prevention in SSA.

\section{Methods}

The protocol for this review was registered on PROSPERO (Registration Number CRD42020186025). This systematic review aimed to combine the results of randomised controlled trials (RCTs) to determine the effectiveness of eHealth interventions for HIV prevention and management in SSA, in comparison to control groups. This review also aimed to compare the effectiveness of interactive and noninteractive eHealth interventions for HIV prevention and management in SSA.

The Theory of Planned Behaviour explains how changes in cognitive outcomes can drive changes in behaviours which in turn can drive improvements in health outcomes [15]. Based on this theory, we decided to use behavioural and biological outcomes as primary measures to determine the effectiveness of interventions, with cognitive and other outcomes as secondary measures.

\section{Inclusion Criteria}

Participants eligible studies were those conducted in SSA countries, as defined by the World Bank [16].

Interventions eHealth interventions were defined as those which were accessible through mobile/Internet/digital means for the purpose of education or behaviour change related to the prevention of sexually transmitted HIV. Interventions could be for the target populations themselves, or sexual health educators (e.g., healthcare workers, community leaders) provided outcomes were measured among the target population. Given that in low-resource settings access to ICT may be limited, we felt that sexual health educators could play a facilitative role in delivering eHealth interventions for HIV prevention and management.

Interventions could be interactive or non-interactive. eHealth interventions were classified as "interactive" when they allowed two-way communication with the user. Interactive interventions required either an automated, programmed system or an active human component to respond to user input.
Study designs randomised controlled trial (RCT) studies published in English from the year 2000.

Comparators minimal intervention (e.g., standard care only) or face-to-face intervention.

Outcomes outcomes were categorised as cognitive, behavioural (HIV prevention or HIV management), biological, or other outcomes related to HIV acquisition. Cognitive outcomes included HIV related knowledge, perceived HIV-related stigma, perceived severity of and susceptibility to HIV, self-efficacy (an individual's belief in their own capacity to carry out a behaviour), and intention to carry out HIV prevention behaviours. Prevention-related behavioural outcomes included condom use, HIV testing and counselling (HTC), and voluntary male circumcision. HIV management-related behavioural outcomes included HIV medication adherence, and uptake and retention in healthcare services for HIV care. Biological outcomes included HIV acquisition, HIV viral load, acquisition of opportunistic infections, and acquisition of other sexually transmitted infections. Other outcomes linked to HIV vulnerability were also included such as use of alcohol or drugs and experience of violence or abuse.

\section{Exclusion Criteria}

We excluded studies which aimed to improve condom use for the purpose of contraception rather than STI prevention, studies of HIV prevention in the context of vertical transmission, studies with composite interventions which inextricably combined digital health interventions with other non-digital interventions, studies with digital interventions for health care workers aimed at optimising healthcare delivery and studies with digital data collection/remote monitoring interventions with no education or behaviour change components.

\section{Search Strategy}

The search focused on eHealth interventions for education or behaviour change aimed at preventing the sexual transmission of HIV in SSA. The following databases were searched on the 5 June 2020 using a tailored search strategy for each: Medline, Embase, Web of Science, Pan-African Clinical Trials Registry, Cochrane Library, PsycInfo. Search strategies for all databases are listed in Supplementary Material 1. The search terms fell into three concepts: "HIV", "eHealth interventions", "Sub-Saharan Africa". The core literature was in the intersection between the three concepts to include eHealth technologies for HIV prevention in SSA. 


\section{Screening}

Papers were screened using Mendeley software and duplicate items were removed using Mendeley's de-duplication tool. One researcher screened the titles, abstracts and full texts for eligibility. $20 \%$ of all papers screened by title and abstract and all papers screened by full text were reviewed by a second researcher. A third reviewer was consulted, where necessary, to reach a consensus. Eligibility was determined based on pre-defined criteria for inclusion and exclusion.

\section{Data Extraction}

One researcher recorded author names, study locations and main findings from the included papers in Excel. All extracted data were independently reviewed by a second researcher to ensure all relevant information was captured accurately.

\section{Data Analysis}

Meta-analyses were undertaken in Cochrane's Review Manager software version 5.4. Conceptually similar outcomes were grouped into the following four categories for analysis: HIV-related cognitive outcomes, HIV prevention behaviours, HIV management behaviours, and biological outcomes. We grouped diverse outcomes into conceptually similar groups, in order to evaluate the effect of eHealth interventions on the outcomes targeted by specific interventions.

When multiple outcomes within the same conceptual group were reported in any one study, one outcome was selected to be included in the analysis. Outcomes were prioritised for selection according to the following criteria: the study's primary outcome, outcomes related to the key aim of the intervention, HIV testing outcomes, condom-use outcomes, objectively measured outcomes, and findings from the longest follow-up period. We sought to evaluate the effect of eHealth interventions on biological outcomes, via their ability to change behaviours related to HIV prevention and management. Behavioural and biological outcomes were therefore the main (primary) outcomes of interest in this review, and these findings were meta-analysed. Findings relating to precursors of behaviour change (cognitive and other outcomes) are also presented.

For meta-analysis, odds ratios (OR) were calculated where possible for each included outcome using the number of events in the intervention and control arms. In some instances means and standard deviations were reported, and standardised mean differences were calculated and re-expressed as ORs following guidance in the Cochrane Handbook [17]. The generic inverse variance method was used to include such data. Where data were not reported for the selected outcome measure, authors were contacted.
ORs in each outcome category were then pooled using a random-effects model, which allows outcomes measured using different scales to be combined. Sub-group analyses were undertaken to determine any differences in the effectiveness of interactive (with and without an active human component) and non-interactive eHealth interventions. The characteristics used for sub-group analysis were specified in advance. The Higgins $\mathrm{I}^{2}$ statistic was used to assess heterogeneity. An $\mathrm{I}^{2}$ statistic of $0 \%, 1-30 \%, 31-50 \%$ and $>50 \%$ indicated no, minimal, moderate and substantial heterogeneity respectively [17]. All calculations were checked by a second researcher.

\section{Risk of Bias and Quality Assessment}

The Cochrane Risk of Bias Tool was used to determine risk of bias in the RCTs. Where there was disagreement between the two researchers who independently reviewed screening, data extraction, and calculations, this was resolved by consulting a third researcher. Studies with retention $>80 \%$ were classed as having low risk of attrition bias. Where metaanalyses contained $>10$ studies, funnel plot asymmetry was used to assess the risk of publication bias across included RCTs [18].

\section{Results}

A total of 4210 unique records were screened by title and abstract, and full texts of 95 potentially eligible papers were screened, resulting in 25 studies being included (see PRISMA flow diagram, Fig. 1) [19].

\section{Characteristics of Included Studies}

Study characteristics of the 25 RCTs included are summarised in Table 1. Studies were undertaken in 10 of the 48 SSA countries, with 8 in Kenya, 7 in Uganda and 5 in South Africa [16]. 14 Studies evaluated interactive eHealth interventions and 12 studies evaluated non-interactive eHealth interventions. Non-interactive eHealth interventions included one-way SMS providing HIV-related information as well as motivational messages and appointment reminders (see intervention characteristics in Supplementary Material 2). Interactive eHealth interventions without an active human component included smartphone games and internetbased programmes, whilst interactive eHealth interventions with an active human component included two-way SMS and mobile phone delivered counselling services. Interventions were delivered alongside standard care.

Control groups received minimal interventions in 24 RCTs (i.e., e.g., standard care only). One RCT compared an eHealth intervention with a face-to-face comparator in 


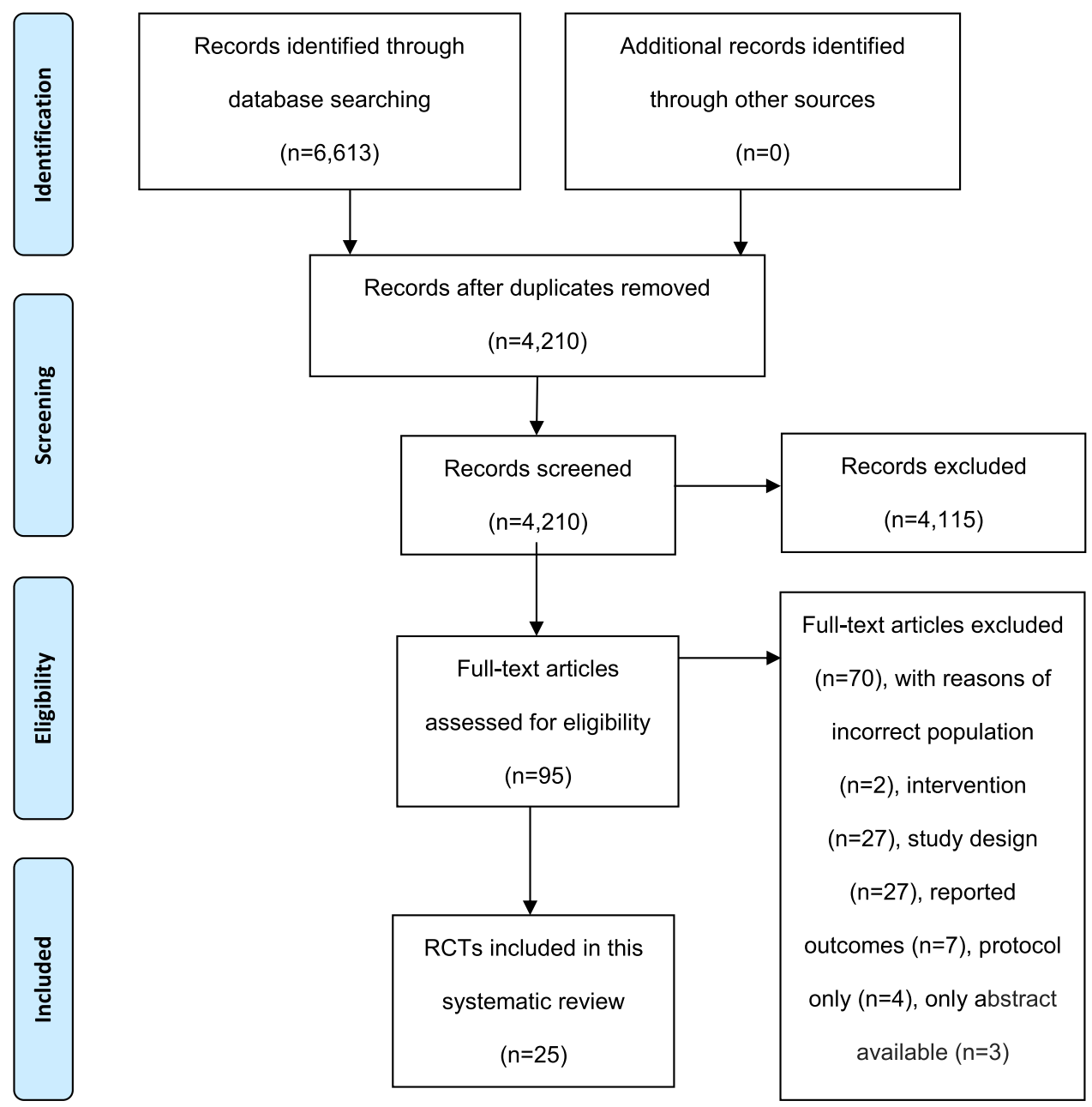

Fig. 1 PRISMA flow diagram [19]

which participants received in-person reminders to improve retention in an HIV vaccine efficacy trial.

Follow-up data collection ranged from immediate to 18 months and sample size ranged from 50 to 1783 . Over half of the studies included $(n=13)$ were conducted solely among people living with HIV. 4 Studies targeted school students and 2 studies targeted high-incidence populations (one among truck drivers and sex workers and the other among people with alcohol use problems).

\section{Quality Assessment}

Table 2 summarises the quality of the 25 RCTs determined using the Cochrane Risk of Bias tool. 17 of the studies were judged "low risk of bias" regarding random sequence generation and 10 regarding allocation concealment. 22 Studies either did not report blinding of participants/personnel or were unblinded; however, given the nature of the interventions this was unsurprising. 19 of the 25 studies had low risk of attrition bias. 5 Studies were at risk of reporting bias as not all outcomes appeared to be analysed and reported in line with a pre-specified plan.

\section{Effectiveness of eHealth Interventions for HIV Prevention and Management}

We included data from 25 RCTs, which randomised a total of 15,343 participants: 2356 were randomised to interactive interventions, 5530 to non-interactive interventions and 5808 to controls.

\section{Can eHealth Technologies Improve HIV Prevention Behaviours?}

6 Studies reported outcomes related to HIV prevention behaviours [20-22, 31, 37, 43]. Behaviours included condom use, attendance for HIV testing and counselling, and uptake of medical male circumcision. Meta-analysis showed that the odds of engaging in HIV prevention behaviours were 
Table 1 Characteristics of included studies

\begin{tabular}{|c|c|c|c|c|c|c|}
\hline Study author, year & Country & Intervention type & Follow up & $\begin{array}{l}\text { Mean par- } \\
\text { ticipant age } \\
\text { (years) }\end{array}$ & $\begin{array}{l}\text { Sample characteris- } \\
\text { tics (size) }\end{array}$ & $\begin{array}{l}\text { Outcomes included in } \\
\text { this review }\end{array}$ \\
\hline Barnabas, 2016 [20] & $\begin{array}{l}\text { South Africa, } \\
\text { Uganda }\end{array}$ & $\begin{array}{l}\text { SMS reminders with } \\
\text { phone call }^{\mathrm{a}}\end{array}$ & 3 months & Not given & $\begin{array}{l}\text { HIV-negative } \\
\text { uncircumcised men } \\
(518)\end{array}$ & $\begin{array}{l}\text { Uptake of medical } \\
\text { male circumcision }\end{array}$ \\
\hline De Tolly, 2011 [21] & South Africa & One-way SMS ${ }^{\mathrm{a}}$ & 3 weeks & Not given & $\begin{array}{l}\text { General population } \\
\text { (2553) }\end{array}$ & $\begin{array}{l}\text { Uptake of HIV testing } \\
\text { and counselling }\end{array}$ \\
\hline Govender, 2019 [22] & $\begin{array}{l}\text { South Africa, Zim- } \\
\text { babwe, Mozam- } \\
\text { bique }\end{array}$ & One-way SMS ${ }^{\mathrm{a}}$ & 6 months & Not given & $\begin{array}{l}\text { Truck drivers and } \\
\text { sex workers (1783) }\end{array}$ & $\begin{array}{l}\text { Uptake of HIV testing; } \\
\text { HIV related knowl- } \\
\text { edge, self-efficacy } \\
\text { and attitudes }\end{array}$ \\
\hline Haberer, 2016 [23] & Uganda & One-way SMS ${ }^{\mathrm{a}}$ & 9 months & Not given & $\begin{array}{l}\text { HIV-positive adults } \\
\text { (62) }\end{array}$ & $\begin{array}{l}\text { Adherence to ART; } \\
\text { viral suppression }\end{array}$ \\
\hline Harder, 2019 [24] & Kenya & $\begin{array}{l}\text { Phone-delivered } \\
\text { motivational } \\
\text { interviewing }\end{array}$ & 6 months & 38 & $\begin{array}{l}\text { Adults with alcohol } \\
\text { use problems (300) }\end{array}$ & Alcohol use \\
\hline Haruna, 2018 [25] & Tanzania & $\begin{array}{l}\text { Sexual health game- } \\
\text { based programme }\end{array}$ & 1 week & 14.1 & $\begin{array}{l}\text { Adolescent school } \\
\text { students (120) }\end{array}$ & HIV-related knowledge \\
\hline $\begin{array}{l}\text { Joseph Davey, } 2016 \\
\text { [26] }\end{array}$ & Mozambique & One-way SMS ${ }^{\mathrm{a}}$ & 12 months & Not given & $\begin{array}{l}\text { HIV-positive adults } \\
\quad(830)\end{array}$ & Retention in ART care \\
\hline $\begin{array}{l}\text { Kalichman, } 2019 \\
\text { [27] }\end{array}$ & South Africa & $\begin{array}{l}\text { Phone delivered } \\
\text { counselling } \\
\text { sessions }^{\mathrm{a}}\end{array}$ & 2 weeks & 34 & $\begin{array}{l}\text { HIV-positive patients } \\
\quad(50)\end{array}$ & $\begin{array}{l}\text { Adherence to ART; } \\
\text { HIV related attitudes }\end{array}$ \\
\hline Kiwanuka, 2018 [28] & Uganda & $\begin{array}{l}\text { SMS with phone } \\
\text { call }^{\text {b }}\end{array}$ & 18 months & Not given & $\begin{array}{l}\text { HIV-negative per- } \\
\text { sons }(662)\end{array}$ & $\begin{array}{l}\text { Retention in HIV vac- } \\
\text { cine trial }\end{array}$ \\
\hline Kurth, 2019 [29] & Kenya & $\begin{array}{l}\text { Internet-based coun- } \\
\text { selling programme }\end{array}$ & 9 months & 37.5 & $\begin{array}{l}\text { HIV-positive adults } \\
\text { (236) }\end{array}$ & $\begin{array}{l}\text { Adherence to ART; } \\
\text { HIV viral suppres- } \\
\text { sion }\end{array}$ \\
\hline Lapinski, 2008 [30] & Nigeria & $\begin{array}{l}\text { Film about HIV- } \\
\text { related stigma }\end{array}$ & Immediate & 27.6 & $\begin{array}{l}\text { General population } \\
\text { (100) }\end{array}$ & HIV related attitudes \\
\hline Leiby, 2016 [31] & Zambia & One-way SMS ${ }^{\mathrm{a}}$ & 6 months & Not given & $\begin{array}{l}\text { Uncircumcised men } \\
\text { (1652) }\end{array}$ & $\begin{array}{l}\text { Uptake of medical } \\
\text { male circumcision }\end{array}$ \\
\hline Lester, 2010 [32] & Kenya & $\begin{array}{l}\text { Two-way SMS with } \\
\text { phone call }^{\mathrm{a}}\end{array}$ & 12 months & 36.7 & $\begin{array}{l}\text { HIV-positive adults } \\
\text { (538) }\end{array}$ & $\begin{array}{l}\text { Adherence to ART; } \\
\text { HIV viral suppres- } \\
\text { sion }\end{array}$ \\
\hline $\begin{array}{l}\text { Linnemayr, } 2017 \\
\text { [33] }\end{array}$ & Uganda & $\begin{array}{l}\text { One- and two-way } \\
\text { SMS }^{\mathrm{a}}\end{array}$ & 12 months & 18.3 & $\begin{array}{l}\text { HIV-positive youth } \\
\text { (332) }\end{array}$ & Adherence to ART \\
\hline $\begin{array}{l}\text { MacCarthy, } 2020 \\
\text { [34] }\end{array}$ & Uganda & One-way SMS ${ }^{\mathrm{a}}$ & 36 weeks & Not given & $\begin{array}{l}\text { HIV-positive youth } \\
\text { (179) }\end{array}$ & Adherence to ART \\
\hline $\begin{array}{l}\text { Mbuagbaw, } 2012 \\
\text { [35] }\end{array}$ & Cameroon & $\begin{array}{l}\text { SMS with phone } \\
\text { call }^{\mathrm{a}}\end{array}$ & 6 months & 40.2 & $\begin{array}{l}\text { HIV-positive adults } \\
\text { (200) }\end{array}$ & $\begin{array}{l}\text { Adherence to ART; } \\
\text { absence of opportun- } \\
\text { istic infections }\end{array}$ \\
\hline Nsagha, 2016 [36] & Cameroon & One-way SMS ${ }^{\mathrm{a}}$ & 1 month & 38.8 & $\begin{array}{l}\text { HIV-positive adults } \\
\text { (90) }\end{array}$ & Adherence to ART \\
\hline Odeny, 2014 [37] & Kenya & One-way SMS ${ }^{\mathrm{a}}$ & 42 days & Not given & $\begin{array}{l}\text { Adult men who } \\
\text { had undergone } \\
\text { male circumcision } \\
\text { (1200) }\end{array}$ & $\begin{array}{l}\text { Abstinence from sex } \\
\text { post circumcision }\end{array}$ \\
\hline $\begin{array}{l}\text { Pop-Eleches, } 2011 \\
\text { [38] }\end{array}$ & Kenya & One-way SMS ${ }^{\mathrm{a}}$ & 48 weeks & 36.3 & $\begin{array}{l}\text { HIV-positive adults } \\
\text { (428) }\end{array}$ & Adherence to ART \\
\hline Reid, 2017 [39] & Botswana & One-way SMS ${ }^{\mathrm{a}}$ & 6 months & 41.1 & $\begin{array}{l}\text { HIV-positive adults } \\
\quad(108)\end{array}$ & $\begin{array}{l}\text { ART pharmacy visit } \\
\text { attendance; CD4 cell } \\
\text { count }\end{array}$ \\
\hline $\begin{array}{l}\text { Van der Kop, } 2018 \\
\text { [40] }\end{array}$ & Kenya & $\begin{array}{l}\text { SMS with phone } \\
\text { call }^{\mathrm{a}}\end{array}$ & 14 months & 33.7 & $\begin{array}{l}\text { HIV-positive adults } \\
\text { (700) }\end{array}$ & Retention in HIV care \\
\hline
\end{tabular}


Table 1 (continued)

\begin{tabular}{|c|c|c|c|c|c|c|}
\hline Study author, year & Country & Intervention type & Follow up & $\begin{array}{l}\text { Mean par- } \\
\text { ticipant age } \\
\text { (years) }\end{array}$ & $\begin{array}{l}\text { Sample characteris- } \\
\text { tics (size) }\end{array}$ & $\begin{array}{l}\text { Outcomes included in } \\
\text { this review }\end{array}$ \\
\hline Venter, 2019 [41] & South Africa & $\begin{array}{l}\text { Educational app with } \\
\text { reminders }^{\mathrm{a}}\end{array}$ & 8 months & Not given & $\begin{array}{l}\text { HIV-positive adults } \\
\text { (353) }\end{array}$ & $\begin{array}{l}\text { Linkage to care for } \\
\text { HIV; HIV viral sup- } \\
\text { pression }\end{array}$ \\
\hline Winskell, 2018 [42] & Kenya & Smartphone game ${ }^{a}$ & 6 weeks & 12.7 & $\begin{array}{l}\text { Pre-adolescent } \\
\text { school students } \\
(60)\end{array}$ & $\begin{array}{l}\text { HIV related knowl- } \\
\text { edge, self-efficacy, } \\
\text { attitudes, and inten- } \\
\text { tions }\end{array}$ \\
\hline Ybarra, 2013 [43] & Uganda & $\begin{array}{l}\text { Internet-based sexual } \\
\text { health programme }\end{array}$ & 6 months & 16.1 & $\begin{array}{l}\text { Secondary school } \\
\text { students }(366)\end{array}$ & Condom use \\
\hline Ybarra, 2015 [44] & Uganda & $\begin{array}{l}\text { Internet-based sexual } \\
\text { health programme }\end{array}$ & 6 months & 16.1 & $\begin{array}{l}\text { Adolescent school } \\
\text { students }(366)\end{array}$ & $\begin{array}{l}\text { HIV related knowl- } \\
\text { edge, self-efficacy, } \\
\text { attitudes, and inten- } \\
\text { tions }\end{array}$ \\
\hline
\end{tabular}

${ }^{a}$ Minimal intervention control

${ }^{b}$ Face-to-face control

not significantly higher among participants who received an eHealth intervention compared with minimal intervention control (OR 1.02; 95\% CI 0.78-1.34; $\mathrm{Z}=0.17$; $\mathrm{p}=0.86$ ) (Fig. 2). Overall, substantial heterogeneity was present $\left(\mathrm{I}^{2}=78 \% ; \chi^{2}=45.73 ; \mathrm{p}<0.00001\right)$.

Sub-group analysis demonstrated that one interactive eHealth intervention with an active human component significantly improved HIV prevention behaviours whereas eHealth interventions without an active human component (2 study arms) and non-interactive eHealth interventions (8 study arms) did not. Significant sub-group differences were found $\left(\chi^{2}=19.94 ; \mathrm{p}<0.0001\right)$.

\section{Can eHealth Technologies Improve HIV Management Behaviours?}

13 Studies reported impacts on behavioural outcomes related to the management of HIV and compared eHealth interventions with minimal intervention control groups. Behavioural outcomes included adherence to ART, attendance at pharmacy visits, and linkage to and retention in HIV care. Two of these studies reported no significant differences in behaviour between study arms, but the data provided in these papers was not in a suitable format for analysis and was not provided by authors upon request [29, 34]. Meta-analysis of 11 studies [23, 26, 27, 32, 33, 35, 36, 38-41] showed that the odds of engaging in behaviours for HIV management were $21 \%$ higher among those in the intervention group compared with control (OR 1.21; 95\% CI 1.05-1.40; $\mathrm{Z}=2.67$; $\mathrm{p}=0.008$ ) (Fig. 3). Overall, minimal heterogeneity was present $\left(I^{2}=15 \% ; \chi^{2}=17.75 ; p=0.28\right)$. There was no evidence of funnel plot asymmetry, indicating a low risk of publication bias across these studies.
In sub-group analysis, interactive eHealth interventions with active human components (5 studies) and non-interactive eHealth interventions (6 studies) led to a statistically significant improvement in HIV management behaviours, but an interactive eHealth intervention without an active human component (1 study) did not. Overall, however, sub-group differences were not statistically significant $\left(\chi^{2}=3.56 ; \mathrm{p}=0.17\right)$.

One RCT was not included in meta-analyses as it was the only study that had a face-to-face control [28]. This study reported significantly worse study retention in the eHealth intervention arm (where participants received short SMS and phone call visit reminders) compared with control (where participants received face-to-face visit reminders) $(\mathrm{p}=0.021)$.

\section{Can eHealth Technologies Improve HIV-Related Biological Outcomes?}

6 Studies reported HIV-related biological outcomes [23, 29, 32, 35, 39, 41]. Outcomes included proportion of participants virally suppressed and with no new opportunistic infections, and mean CD4 cell count. Meta-analysis showed that the odds of improved biological outcomes were not significantly higher among those who received an eHealth intervention compared with minimal intervention control (OR 1.17; 95\% CI 0.89-1.54; $\mathrm{Z}=1.10 ; \mathrm{p}=0.27$ ) (Fig. 4). Overall, no heterogeneity was present $\left(\mathrm{I}^{2}=0 \% ; \chi^{2}=5.60\right.$; $\mathrm{p}=0.47$ ).

Sub-group analysis demonstrated that compared with minimal intervention controls the impact of eHealth interventions on HIV-related biological outcomes was not significant for any of the three sub-groups: interactive eHealth 
Table 2 Quality appraisal of the studies

$\begin{array}{ccccc}\text { Random } & \text { Allocation } & \text { Participant and } & \text { Attrition } & \text { Selective } \\ \text { allocation } & \text { concealment } & \text { personnel } & \text { bias } & \text { reporting } \\ & & \text { blinding } & & \end{array}$

\begin{tabular}{|c|c|c|c|c|c|}
\hline Barnabas 2016 & $\checkmark$ & $?$ & $x$ & $\checkmark$ & $\checkmark$ \\
\hline De Tolly 2011 & $?$ & ? & $?$ & $x$ & $\checkmark$ \\
\hline Govender 2019 & $?$ & $\checkmark$ & $?$ & $x$ & $x$ \\
\hline Haberer 2016 & $\checkmark$ & $?$ & $x$ & $\checkmark$ & $\checkmark$ \\
\hline Harder 2019 & $?$ & ? & $?$ & $\checkmark$ & $\checkmark$ \\
\hline Haruna 2018 & $?$ & $?$ & $?$ & $\checkmark$ & $\checkmark$ \\
\hline Joseph Davey 2016 & $\checkmark$ & $\checkmark$ & $\checkmark$ & $\times$ & $\checkmark$ \\
\hline Kalichman 2019 & $\checkmark$ & $?$ & $\checkmark$ & $\checkmark$ & $\checkmark$ \\
\hline Kiwanuka 2018 & $\checkmark$ & $\checkmark$ & $x$ & $x$ & $\checkmark$ \\
\hline Kurth 2019 & $x$ & $x$ & $?$ & $\checkmark$ & $x$ \\
\hline Lapinski 2008 & $x$ & $?$ & $?$ & $\checkmark$ & $x$ \\
\hline Leiby 2016 & $?$ & $?$ & $?$ & $x$ & $\checkmark$ \\
\hline Lester 2010 & $\checkmark$ & $\checkmark$ & $x$ & $x$ & $\checkmark$ \\
\hline Linnemayr 2017 & $\checkmark$ & $?$ & $x$ & $\checkmark$ & $\checkmark$ \\
\hline MacCarthy 2020 & $\checkmark$ & $\checkmark$ & $x$ & $\checkmark$ & $\checkmark$ \\
\hline Mbuagbaw 2012 & $\checkmark$ & $\checkmark$ & $\checkmark$ & $\checkmark$ & $\checkmark$ \\
\hline Nsagha 2016 & $\checkmark$ & $?$ & $?$ & $\checkmark$ & ? \\
\hline Odeny 2014 & $\checkmark$ & $\checkmark$ & $x$ & $\checkmark$ & $\checkmark$ \\
\hline Pop-Eleches 2011 & $\checkmark$ & ? & $?$ & $\checkmark$ & $\checkmark$ \\
\hline Reid 2017 & $?$ & $\checkmark$ & $x$ & $\checkmark$ & $x$ \\
\hline Van der Kop 2018 & $\checkmark$ & $\checkmark$ & $x$ & $\checkmark$ & $\checkmark$ \\
\hline Venter 2019 & $\checkmark$ & $?$ & $?$ & $\checkmark$ & $\checkmark$ \\
\hline Winskell 2018 & $\checkmark$ & $\checkmark$ & $\times$ & $\checkmark$ & $\checkmark$ \\
\hline Ybarra 2013 & $\checkmark$ & $?$ & $x$ & $\checkmark$ & $x$ \\
\hline Ybarra 2015 & $\checkmark$ & ? & $x$ & $\checkmark$ & $\checkmark$ \\
\hline
\end{tabular}

Risk of bias: $\checkmark=$ low; ? uncertain; $X=$ high 
Experimental Control Odds Ratio

Events Total Events Total Weight M-H, Random, $95 \% \mathrm{Cl}$

Odds Ratio

5.1.1 Interactive eHealth intervention with acive human component

$\begin{array}{lcccccr}\text { Barnabas 2016 (1) } & 137 & 284 & 62 & 224 & 10.5 \% & 2.44[1.68,3.54] \\ \text { Subtotal (95\% Cl) } & & \mathbf{2 8 4} & & \mathbf{2 2 4} & \mathbf{1 0 . 5 \%} & \mathbf{2 . 4 4}[\mathbf{1 . 6 8}, \mathbf{3 . 5 4}] \\ \text { Total events } & 137 & & 62 & & & \end{array}$

Heterogeneity: Not applicable

Test for overall effect: $Z=4.66(P<0.00001)$

\subsubsection{Interactive eHealth intervention without active human component}

\begin{tabular}{|c|c|c|c|c|c|c|}
\hline Ybarra 2013 a (2) & 68 & 83 & 147 & 171 & $6.9 \%$ & $0.74[0.37,1.50]$ \\
\hline $\begin{array}{l}\text { Ybarra } 2013 \mathrm{~b}(3) \\
\text { Subtotal }(95 \% \mathrm{Cl})\end{array}$ & 74 & $\begin{array}{r}85 \\
168\end{array}$ & 147 & $\begin{array}{l}171 \\
342\end{array}$ & $\begin{array}{r}6.4 \% \\
13.3 \%\end{array}$ & $\begin{array}{l}1.10[0.51,2.36] \\
0.89[0.53,1.49]\end{array}$ \\
\hline \multicolumn{7}{|c|}{$\begin{array}{l}\text { Heterogeneity: } \mathrm{Tau}^{2}=0.00 ; \mathrm{Chi}^{2}=0.55, \mathrm{df}=1(P=0.46) ; I^{2}=0 \% \\
\text { Test for overall effect: } Z=0.45(P=0.65)\end{array}$} \\
\hline \multicolumn{7}{|c|}{ 5.1.3 Non-interactive eHealth intervention } \\
\hline De Tolly 2011 a (4) & 120 & 252 & 224 & 403 & $11.2 \%$ & $0.73[0.53,1.00]$ \\
\hline De Tolly 2011 b (5) & 143 & 320 & 224 & 403 & $11.4 \%$ & $0.65[0.48,0.87]$ \\
\hline De Tolly 2011 c (6) & 130 & 249 & 224 & 403 & $11.1 \%$ & $0.87[0.64,1.2$ \\
\hline De Tolly 2011 d (7) & 154 & 271 & 224 & 403 & $11.2 \%$ & $1.05[0.77,1.4$ \\
\hline Govender 2019 (8) & 323 & 375 & 265 & 341 & $10.3 \%$ & $1.78[1.21,2.6$ \\
\hline Leiby 2016 a (9) & 10 & 533 & 8 & 513 & $5.1 \%$ & $1.21[0.47,3.08]$ \\
\hline Leiby 2016 b (10) & 6 & 495 & 8 & 513 & $4.3 \%$ & $0.77[0.27,2.25]$ \\
\hline $\begin{array}{l}\text { Odeny } 2014(11) \\
\text { Subtotal }(95 \% \mathrm{CI})\end{array}$ & 352 & $\begin{array}{r}491 \\
2986\end{array}$ & 369 & $\begin{array}{r}493 \\
3472\end{array}$ & $\begin{array}{l}11.5 \% \\
76.2 \%\end{array}$ & $\begin{array}{l}0.85[0.64,1.13] \\
0.92[0.73,1.16]\end{array}$ \\
\hline Total events & 1238 & & 1546 & & & \\
\hline
\end{tabular}

Heterogeneity: $\mathrm{Tau}^{2}=0.06 ; \mathrm{Chi}^{2}=20.09, \mathrm{df}=7(\mathrm{P}=0.005) ; \mathrm{I}^{2}=65 \%$

Test for overall effect: $Z=0.69(P=0.49)$

Total $(95 \% \mathrm{Cl})$

3438

$4038 \quad 100.0 \%$

Total events 1517

1902

Heterogeneity: $\mathrm{Tau}^{2}=0.14 ; \mathrm{Chi}^{2}=45.73, \mathrm{df}=10(\mathrm{P}<0.00001) ; \mathrm{I}^{2}=78 \%$

Test for overall effect: $Z=0.17(P=0.86)$

Test for subgroup differences: $\mathrm{Chi}^{2}=19.94, \mathrm{df}=2(\mathrm{P}<0.0001), \mathrm{I}^{2}=90.0 \%$

$1.02[0.78,1.34]$

Footnotes

(1) Uptake of medical male circumcision

(2) Proportion that reported protected sex or no vaginal sex (intervention no booster)

(3) Proportion that reported protected sex or no vaginal sex (intervention + booster)

(4) Proportion that attended HTC (3 motivational messages)

(5) Proportion that attended HTC (10 motivational messages)

(6) Proportion that attended HTC (3 informational messages)

(7) Proportion that attended HTC (10 informational messages)

(8) Proportion that tested for HIV in the previous 6 months

(9) Verified voluntary medical male circumcision uptake (conventional arm)

(10) Verified voluntary medical male circumcision uptake (tailored arm)

(11) Proportion that abstained from sex in the first 42 days post circumcision

Fig. 2 Forest plot: behaviours related to HIV prevention

interventions with an active human component (2 studies), interactive eHealth interventions without an active human component (2 studies) and non-interactive eHealth interventions (2 studies). Overall, sub-group differences were not statistically significant $\left(\chi^{2}=0.29 ; \mathrm{p}=0.86\right)$.

\section{Can eHealth Technologies Improve HIV-Related Cognitive and Other Outcomes?}

Cognitive outcomes reported were HIV-related knowledge, self-efficacy, attitudes and intentions. All the 4 studies that reported HIV-related knowledge found that eHealth interventions significantly improved knowledge compared with control [22, 25, 42, 44]. 3 Studies measured self-efficacy: 1 study reported significantly improved self-efficacy [42] and 2 reported no statistically significant differences 


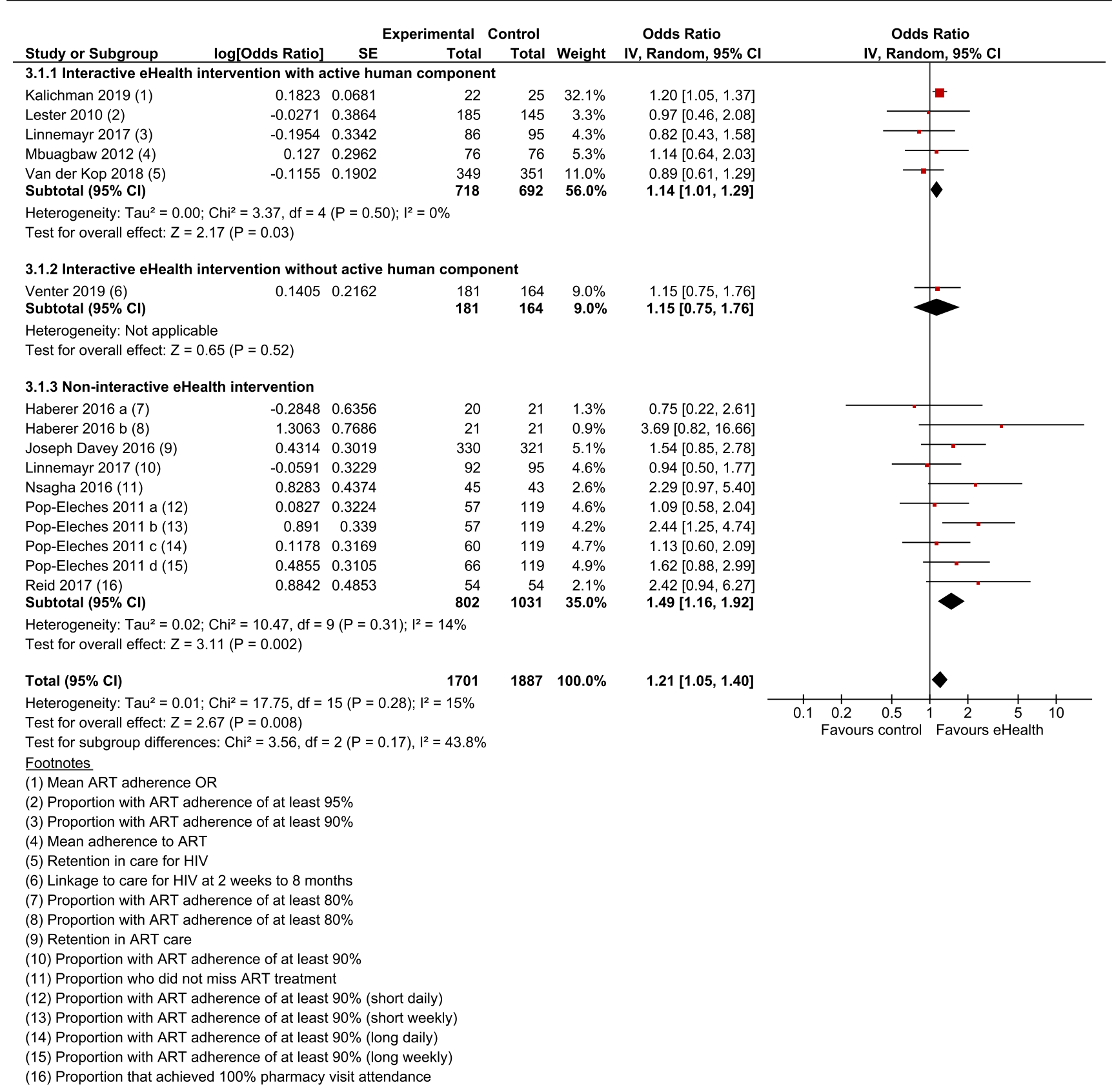

Fig. 3 Forest plot: behaviours related to HIV management

between intervention and control groups [22, 44]. 5 Studies measured attitudes (towards condom use, towards the perceived severity of and susceptibility to HIV, and towards HIV-related stigma); 2 found significant improvements in attitudes [27, 44] and 3 found no statistically significant differences between intervention and control arms [22, 30, 42]. 2 Studies measured intention to carry out HIV prevention behaviours; both found significant improvement among those in the eHealth intervention arm compared with control $[42,44] .1$ Study assessed the impact of eHealth interventions on other outcomes which found that alcohol use was significantly reduced among participants in the intervention arm compared to control [24].

\section{Discussion}

We found that eHealth interventions deployed in SSA increased engagement in HIV management behaviours but did not significantly impact HIV prevention behaviours or HIV-related biological outcomes. eHealth interventions had positive effects on HIV-related knowledge and intentions to 


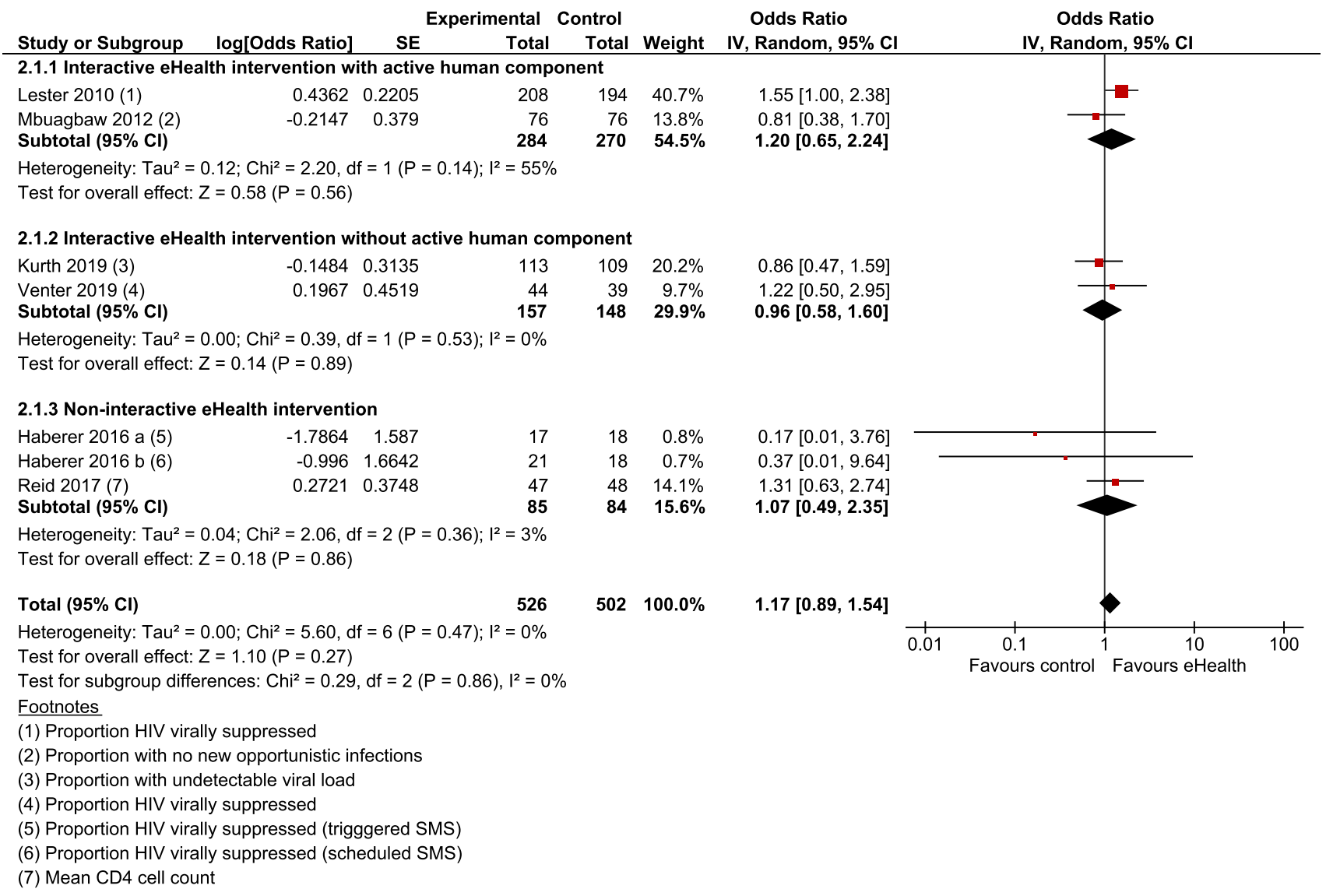

Fig. 4 Forest plot: HIV-related biological outcomes

reduce the likelihood of, or avoid, HIV transmission/acquisition. This review provides good evidence supporting the use of eHealth interventions to improve HIV management behaviours in SSA.

The findings of this systematic review resonate with existing literature. Two other systematic reviews, which include studies from Europe, North America, South America, Africa, Oceania, and Asia, demonstrate the positive impact of eHealth interventions on HIV management behaviours. The first demonstrates the impact of eHealth interventions, predominantly SMS interventions, on adherence to ART and clinic attendance [13]. The second, larger review found similar positive effects, reporting that the odds of improved adherence to ART were more than double (OR; 95\% $\mathrm{CI}=2.15 ; 1.18-3.91)$ among those who received eHealth interventions compared with control [14]. One qualitative study undertaken in Uganda emphasised the importance of developing new habits to augment the impact of eHealth interventions on adherence to ART after intervention withdrawal [45].

We found that eHealth interventions did not significantly improve behaviours related to HIV prevention. This finding is discordant with that of Kemp and Velloza, whose
2018 review found that eHealth interventions can effectively improve uptake of HIV testing [13]. The moderate to significant heterogeneity found among the studies in our meta-analyses indicates that there was large variability in the effect sizes of the individual RCTs, which creates uncertainty in our results. Heterogeneity may have arisen due to differences between studies in participant characteristics/ settings or methodologies [17].

Despite improving HIV management behaviours, our review found no impact on biological outcomes, unlike other global research which demonstrates a significant impact of mHealth on HIV viral load and CD4 cell count [46]. It is possible that this was because the overall sample size was not large enough to detect significant differences between arms. An important consideration is that changes in HIVrelated biological outcomes are often difficult to measure due to the infrequency of events such as HIV acquisition (particularly when studies only have short follow-up periods) and the practical challenges of biological outcome measurement [47].

It is important to capture any differences between interactive and non-interactive interventions as there can be cost implications for interventions which require a greater level 
of provider support, particularly for those with an active human component [48]. Although significant sub-group differences were present in the analysis of HIV prevention behaviours, there were no statistically significant differences found between sub-groups for HIV management behaviours and biological outcomes. These results seem to contradict existing literature which reports that interactive digital interventions tend to have a greater impact than those that are non-interactive [12, 47, 49]. We know, however, that the rate of uptake of new technologies has been slower in SSA due to poorer digital infrastructure and that smartphone ownership in SSA is lower than any other region worldwide [8], which may explain why interactive Internet-based sexual health programmes and smartphone apps/games are not as effective as we would expect based on the findings from other studies.

\section{Strengths and Limitations}

This systematic review is the first to investigate the effectiveness of eHealth interventions for HIV prevention and management in SSA. We included 25 RCTs which are the most appropriate design to assess effectiveness [50]. Included studies were conducted in 10 of SSA's 48 countries, which is likely to reflect differences in HIV research activity within the region, although it is also possible that we missed relevant papers by restricting our review to English language publications. Very few of the included studies were at low risk of any bias, and some study data could not be obtained for inclusion in meta-analyses.

\section{Recommendations for Future Work}

More RCTs on eHealth interventions for HIV prevention and management in SSA are required to provide greater certainty in the evidence of their effectiveness, including measurement of biological outcomes. More qualitative studies are also needed to understand the context and complexity of health needs, and the feasibility and acceptability of widescale eHealth implementation. Despite the fact that SSA experiences around $70 \%$ of all global HIV diagnoses, less than $23 \%$ of all HIV/AIDS research involves participation from researchers in Africa [1, 51]. Within the African continent, research tends to be concentrated in a few countries, predominantly South Africa, Uganda and Kenya [51]. There should be more HIV research in all countries across SSA, with more direct involvement of SSA researchers.

\section{Implications for Practice}

The finding that eHealth interventions can improve behaviours related to HIV management in SSA is tremendously important. Through improving adherence to ART and retention in HIV care, eHealth interventions can be used to minimise the likelihood of people living with HIV transmitting HIV to others and reduce HIV-related morbidity and mortality across SSA [3, 52]. Healthcare systems across SSA face challenges with scare resources, inadequate finances and staff shortages, and low-cost eHealth strategies have huge potential to improve HIV-related outcomes across the region [53].

Supplementary Information The online version contains supplementary material available at https://doi.org/10.1007/s10461-021-03402-w.

Acknowledgements We thank Simon Moore and colleagues at Avert (an international HIV and AIDS charity) for their helpful insights into the HIV epidemic and prevention context in Sub-Saharan Africa and informed suggestions as to the applicability of our findings.

Author Contributions LM: Conceptualisation, Methodology, Searches, Data extraction and analysis, Writing-first draft. CA: Second checking calculations, Writing-review and editing. MD: Second checking screening and data extraction, Writing-review and editing. JB: Conceptualisation, Methodology, Writing-review and editing.

Funding No funding was received to assist with the preparation of this manuscript.

Data Availability No additional data available (common).

Code availability Not applicable.

\section{Declarations}

Conflict of interest The authors have no relevant financial or non-financial interests to disclose.

Ethical Approval Not applicable.

Informed Consent Not applicable.

Consent for Publication Not applicable.

Open Access This article is licensed under a Creative Commons Attribution 4.0 International License, which permits use, sharing, adaptation, distribution and reproduction in any medium or format, as long as you give appropriate credit to the original author(s) and the source, provide a link to the Creative Commons licence, and indicate if changes were made. The images or other third party material in this article are included in the article's Creative Commons licence, unless indicated otherwise in a credit line to the material. If material is not included in the article's Creative Commons licence and your intended use is not permitted by statutory regulation or exceeds the permitted use, you will need to obtain permission directly from the copyright holder. To view a copy of this licence, visit http://creativecommons.org/licenses/by/4.0/.

\section{References}

1. UNAIDS. Global report: UNAIDS report on the global AIDS epidemic 2010. UNAIDS; 2010. https://www.unaids.org/globalrepo 
rt/documents/20101123_GlobalReport_full_en.pdf. Accessed 2 Sep 2020.

2. Dwyer-Lindgren L, Cork MA, Sligar A, et al. Mapping HIV prevalence in sub-Saharan Africa between 2000 and 2017. Nature. 2019;570(7760):189-93.

3. Wringe A, Renju J, Seeley J, Moshabela M, Skovdal M. Bottlenecks to HIV care and treatment in sub-Saharan Africa: a multicountry qualitative study. Sex Transm Infect. 2017;93(Suppl 3):e053172.

4. UNAIDS. The Gap Report. UNAIDS; 2014. https://unaids-test. unaids.org/sites/default/files/unaids/contentassets/documents/ unaidspublication/2014/UNAIDS_Gap_report_en.pdf. Accessed 2 Aug 2020.

5. Holst C, Sukums F, Radovanovic D, Ngowi B, Noll J, Winkler AS. Sub-Saharan Africa - the new breeding ground for global digital health. Lancet Digit Health. 2020;2(4):e160-2.

6. Asongu SA, Nwachukwu JC, Aziz A. Determinants of mobile phone penetration: panel threshold evidence from Sub-Saharan Africa. J Glob Inf Technol Manag. 2018;21(2):81-110.

7. Maloney KM, Bratcher A, Wilkerson R, Sullivan PS. Electronic and other new media technology interventions for HIV care and prevention: a systematic review. J Int AIDS Soc. 2020;23(1):e25439.

8. Silver L, Johnson C. Majorities in Sub-Saharan Africa own mobile phones, but smartphone adoption is modest. 2018. https://www. pewresearch.org/global/2018/10/09/majorities-in-sub-saharanafrica-own-mobile-phones-but-smartphone-adoption-is-modest/. Accessed 12 Jan 2021.

9. Adeloye D, Adigun T, Misra S, et al. Assessing the coverage of e-Health services in Sub-Saharan Africa. Methods Inf Med. 2017;56(3):189-99.

10. Tomlinson M, Rotheram-Borus MJ, Swartz L, Tsai AC. Scaling up mHealth: where is the evidence? PLoS Med. 2013;10(2):e1001382.

11. Bervell B, Al-Samarraie H. A comparative review of mobile health and electronic health utilization in Sub-Saharan African countries. Soc Sci Med. 2019;232:1-16.

12. Bailey JV, Wayal S, Aicken C, et al. Interactive digital interventions for prevention of sexually transmitted HIV: systematic review and meta-analyses. AIDS. 2021;35(4):643-53. https://doi. org/10.1097/QAD.0000000000002780.

13. Kemp CG, Velloza J. Implementation of eHealth interventions across the HIV care cascade: a review of recent research. Curr HIV/AIDS Rep. 2018;15(6):403-13.

14. Daher J, Vijh R, Linthwaite B, et al. Do digital innovations for HIV and sexually transmitted infections work? Results from a systematic review (1996-2017). BMJ Open. 2017;7(11):e017604.

15. Ajzen I. The theory of planned behavior. Organ Behav Hum Decis Process. 1991;50(2):179-211.

16. The World Bank. Sub-Saharan Africa. The World Bank; 2020. https://data.worldbank.org/country/ZG. Accessed 12 Jan 2021.

17. Deeks J, Higgins J, Altman D. Analysing data and undertaking meta-analyses: Cochrane Training. 2020. https://training.cochr ane.org/handbook/current/chapter-10. Accessed 11 May 2020.

18. Sterne JAC, Sutton AJ, Ioannidis JPA, et al. Recommendations for examining and interpreting funnel plot asymmetry in metaanalyses of randomised controlled trials. BMJ. 2011;343:d4002.

19. Moher D, Liberati A, Tetzlaff J, Altman DG, The PRISMA Group. Preferred Reporting Items for Systematic Reviews and Meta-Analyses: the PRISMA statement. PLoS Med. 2009;6(7):e1000097.

20. Barnabas RV, van Rooyen H, Tumwesigye E, et al. Uptake of antiretroviral therapy and male circumcision after communitybased HIV testing and strategies for linkage to care versus standard clinic referral: a multisite, open-label, randomised controlled trial in South Africa and Uganda. Lancet HIV. 2016;3(5):e212-20.
21. De Tolly K, Skinner D, Nembaware V. Investigation into the use of short message services to expand uptake of human immunodeficiency virus testing, and whether content and dosage have impact. Telemed J e-Health Off J Am Telemed Assoc. 2011;18:18-23.

22. Govender K, Beckett S, Masebo W, et al. Effects of a short message service (SMS) intervention on reduction of HIV risk behaviours and improving HIV testing rates among populations located near roadside wellness clinics: a cluster randomised controlled trial in South Africa, Zimbabwe and Mozambique. AIDS Behav. 2019;23(11):3119-28.

23. Haberer JE, Musiimenta A, Atukunda EC, et al. Short message service (SMS) reminders and real-time adherence monitoring improve antiretroviral therapy adherence in rural Uganda. AIDS (Lond Engl). 2016;30(8):1295-300.

24. Harder VS, Musau AM, Musyimi CW, Ndetei DM, Mutiso VN. A randomized clinical trial of mobile phone motivational interviewing for alcohol use problems in Kenya. Addiction. 2020;115(6):1050-60.

25. Haruna H, Hu X, Chu SKW, Mellecker RR, Gabriel G, Ndekao PS. Improving sexual health education programs for adolescent students through game-based learning and gamification. Int $\mathbf{J}$ Environ Res Public Health. 2018;15(9):2027.

26. Joseph Davey D, Nhavoto JA, Augusto O, et al. SMSaúde: evaluating mobile phone text reminders to improve retention in HIV care for patients on antiretroviral therapy in Mozambique. J Acquir Immune Defic Syndr (1999). 2016;73(2):e23-30.

27. Kalichman SC, Mathews C, Banas E, Kalichman MO. Stigma management intervention to improve antiretroviral therapy adherence: Phase-I test of concept trial, Cape Town, South Africa. Glob Public Health. 2019;14(8):1059-74.

28. Kiwanuka N, Mpendo J, Asiimwe S, et al. A randomized trial to assess retention rates using mobile phone reminders versus physical contact tracing in a potential HIV vaccine efficacy population of fishing communities around Lake Victoria, Uganda. BMC Infect Dis. 2018;18(1):591.

29. Kurth AE, Sidle JE, Chhun N, et al. Computer-based counseling program (CARE+ Kenya) to promote prevention and HIV health for people living with HIV/AIDS: a randomized controlled trial. AIDS Educ Prev Off Publ Int Soc AIDS Educ. 2019;31(5):395-406.

30. Lapinski MK, Nwulu P. Can a short film impact HIV-related risk and stigma perceptions? Results from an experiment in Abuja, Nigeria. Health Commun. 2008;23(5):403-12.

31. Leiby K, Connor A, Tsague L, et al. The impact of SMS-based interventions on VMMC uptake in Lusaka Province, Zambia: a randomized controlled trial. J Acquir Immune Defic Syndr (1999). 2016;72(Suppl 4):S264-72.

32. Lester RT, Ritvo P, Mills EJ, et al. Effects of a mobile phone short message service on antiretroviral treatment adherence in Kenya (WelTel Kenya1): a randomised trial. Lancet. 2010;376(9755):1838-45.

33. Linnemayr S, Huang $\mathrm{H}$, Luoto J, et al. Text messaging for improving antiretroviral therapy adherence: no effects after 1 year in a randomized controlled trial among adolescents and young adults. Am J Public Health. 2017;107(12):1944-50.

34. MacCarthy S, Wagner Z, Mendoza-Graf A, et al. A randomized controlled trial study of the acceptability, feasibility, and preliminary impact of SITA (SMS as an Incentive To Adhere): a mobile technology-based intervention informed by behavioral economics to improve ART adherence among youth in Uganda. BMC Infect Dis. 2020;20(1):173.

35. Mbuagbaw L, Thabane L, Ongolo-Zogo P, et al. The Cameroon Mobile Phone SMS (CAMPS) Trial: a randomized trial of text messaging versus usual care for adherence to antiretroviral therapy. PLoS ONE. 2012;7(12):e46909. 
36. Nsagha DS, Lange I, Fon PN, Nguedia Assob JC, Tanue EA. A randomized controlled trial on the usefulness of mobile text phone messages to improve the quality of care of HIV and AIDS patients in Cameroon. Open AIDS J. 2016;10:93-103.

37. Odeny TA, Bailey RC, Bukusi EA, et al. Effect of text messaging to deter early resumption of sexual activity after male circumcision for HIV prevention: a randomized controlled trial. J Acquir Immune Defic Syndr (1999). 2014;65(2):e50-7.

38. Pop-Eleches C, Thirumurthy H, Habyarimana JP, et al. Mobile phone technologies improve adherence to antiretroviral treatment in a resource-limited setting: a randomized controlled trial of text message reminders. AIDS (Lond Engl). 2011;25(6):825-34.

39. Reid MJA, Steenhoff AP, Thompson J, et al. Evaluation of the effect of cellular SMS reminders on consistency of antiretroviral therapy pharmacy pickups in HIV-infected adults in Botswana: a randomized controlled trial. Health Psychol Behav Med. 2017;5(1):101-9.

40. Van der Kop ML, Muhula S, Nagide PI, et al. Effect of an interactive text-messaging service on patient retention during the first year of HIV care in Kenya (WelTel Retain): an openlabel, randomised parallel-group study. Lancet Public Health. 2018;3(3):e143-52.

41. Venter WDF, Fischer A, Lalla-Edward ST, et al. Improving linkage to and retention in care in newly diagnosed HIV-positive patients using smartphones in South Africa: randomized controlled trial. JMIR Mhealth Uhealth. 2019;7(4):e12652.

42. Winskell K, Sabben G, Akelo V, et al. A smartphone gamebased intervention (Tumaini) to prevent HIV among young Africans: pilot randomized controlled trial. JMIR Mhealth Uhealth. 2018;6(8):e10482.

43. Ybarra ML, Bull SS, Prescott TL, Korchmaros JD, Bangsberg DR, Kiwanuka JP. Adolescent abstinence and unprotected sex in CyberSenga, an Internet-based HIV prevention program: randomized clinical trial of efficacy. PLoS ONE. 2013;8(8):e70083.

44. Ybarra ML, Korchmaros JD, Prescott TL, Birungi R. A randomized controlled trial to increase HIV preventive information, motivation, and behavioral skills in Ugandan adolescents. Ann Behav Med Publ Soc Behav Med. 2015;49(3):473-85.
45. Musiimenta A, Atukunda EC, Tumuhimbise W, Haberer JE. Resilience after withdrawing a technology-based medication adherence support intervention from people living with HIV in rural Uganda. AIDS Care. 2018;30(sup5):S89-96.

46. Cooper V, Clatworthy J, Whetham J, EmERGE Consortium. mHealth interventions to support self-management in HIV: a systematic review. Open AIDS J. 2017;11:119-32.

47. Bailey JV, Murray E, Rait G, et al. Interactive computer-based interventions for sexual health promotion. Cochrane Database Syst Rev. 2010. https://doi.org/10.1002/14651858.cd006483. pub2.

48. Bailey J, Mann S, Wayal S. Sexual health promotion for young people delivered via digital media: a scoping review. NIHR Journals Library: Chapter 5, Health economic evaluations in sexual health promotion delivered by digital media. 2015. https://www. ncbi.nlm.nih.gov/books/NBK326979/?report=classic. Accessed 26 Aug 2020.

49. Chiang N, Guo M, Amico KR, Atkins L, Lester RT. Interactive Two-way mHealth interventions for improving medication adherence: an evaluation using the behaviour change wheel framework. JMIR Mhealth Uhealth. 2018;6(4):e87.

50. Hariton E, Locascio JJ. Randomised controlled trials - the gold standard for effectiveness research: study design: randomised controlled trials. BJOG Int J Obstet Gynaecol. 2018;125(13):1716.

51. González-Alcaide G, Menchi-Elanzi M, Nacarapa E, RamosRincón J-M. HIV/AIDS research in Africa and the Middle East: participation and equity in North-South collaborations and relationships. Glob Health. 2020;16(1):83.

52. Okoli C, Van de Velde N, Richman B, et al. Undetectable equals untransmittable $(\mathrm{U}=\mathrm{U})$ : awareness and associations with health outcomes among people living with HIV in 25 countries. Sex Transm Infect. 2021;97:18-26.

53. Aranda-Jan CB, Mohutsiwa-Dibe N, Loukanova S. Systematic review on what works, what does not work and why of implementation of mobile health (mHealth) projects in Africa. BMC Public Health. 2014;14(1):188.

Publisher's Note Springer Nature remains neutral with regard to jurisdictional claims in published maps and institutional affiliations. 